\title{
Effects of Forest Healing Program on Cultural Adaptation Stress and Psychological Wellbeing of Foreign Workers
}

\author{
Han-Won Kim ${ }^{1}$, Won-Sop Shin ${ }^{2 *}$, and Cheoul-Soon Jang ${ }^{1}$ \\ ${ }^{1}$ Graduate Department of Forest Therapy, Chungbuk National University, Cheongju 28644, Korea \\ ${ }^{2}$ Department of Forestry, Chungbuk National University, Cheongju 28644, Korea
}

\section{ABSTRACT}

As the number of foreign workers increases in Korea, people's interest in their adaptation to this society has also increased. The purpose of this study was to investigate the effect of a forest-healing program on foreign workers' cultural adaptation stress and psychological wellbeing. The experiment was conducted in a recreation forest located in Hongcheon-gun, Gangwon-do. The subjects who participated in this study included 40 foreign workers (16 males, 24 females). The experiment was conducted for three days and two nights from June 26 to 28, 2019. The forest healing program was composed of activities that can enhance immunity and restore physical and mental health by utilizing various healing environmental factors such as landscapes, sunlight, anion and oxygen in forest. The independent sample t-test was conducted to examine changes in cultural adaptation stress and psychological well-being before and after the program. The collected data were analyzed using SPSS 18.0 program. As a result, cultural adaptation stress was statistically significantly reduced in sub-factors including discrimination experience, language conflict and legal status. Psychological wellbeing improved in all the surveyed sub-factors such as self-confidence, ego, immersion, and joy. The results of this study are expected to be used as basic data for reducing the stress foreign workers experience from cultural adaptation and restoring their psychological wellbeing in the future.

Keywords: domestic workers, forest environment, forest therapy

\section{Introduction}

The number of foreigners who are living in Korea for various purposes has increased. Not only Korea, but also many countries around the world have seen significant changes in the composition of labor force, which has made individuals, who had to adapt themselves to their own culture and other new cultures, experience mental and physical stress due to cultural shock. The difficulties that foreign workers, as an immigrant, face in living in Korea have doubled due to differences between what they expected from their life in Korea and the actual life (Hendriks and Bartram, 2016; Liu and Zhang, 2015). As such, the increasing number of foreigners staying in Korea has also resulted in several social problems (Han and Choi, 2011). The number of foreigners who committed crimes in Korea has gradually increased (Korean National Police Agency, 2016), and married immigrants were reported to have difficulties in settling in Korea due to various issues, such as conflicts with their spouses, financial difficulties, difficulties in communication, and conflicts between cultures like different lifestyles (Kim, 2012). People have to face a process of cultural adaptation whether the environment of adaptation is good or bad whenever they enter another society with different culture (Berry, 1997). As they try to adapt themselves to a new environment, they are affected from different sides, whether it is society or individuals, by absence of social support, and pressure they feel in achieving men-

Received: September 18, 2019, Revised: September 25, 2019, Accepted: October 8, 2019

First author: Han-Won Kim, unicon4999@hanmail.net, (1) https://orcid.org/0000-0002-1231-5056

*Corresponding author: Won-Sop Shin, shinwon@ @ chungbuk.ac.kr, (10) https://orcid.org/0000-0002-5665-1502 
tal goals. From a macroscopic perspective, social factors such as the level of social support, and strategies suggested by the government for cultural adaptation affect stress from cultural adaptation, and from a microscopic perspective, people are affected by personal factors such as personality, educational background, previous experience of multiple cultures and language proficiency.

Stress from cultural adaptation is a stress that individuals experience due to several factors in adapting themselves from their own cultural background to a new culture (Berry et al., 1987), or a stress that they experience in the process of settling and cultural adaptation due to various environmental factors such as financial difficulties and cultural conflicts (Berry, 1997). Foreign workers who immigrate from a different cultural area, a familiar environment, mostly experience stress from cultural adaptation due to discrimination, identity confusion, and a sense of loss as they lose their social and economic position, and their support system (Berry, 1984; Lee et al., 2009). Those who had a low level of stress from cultural adaptation were reported to show more prosocial behaviors such as helping and cooperating with others and voluntarily giving help to others (Kim and Kim, 2011). Those who have a high level of stress tend to show high crime and accident rates (Park and Lim, 2000). Stress that foreigners experience in Korea during cultural adaptation can be recognized as one of the causes of these social problems. Their attempts to effectively adapt themselves to an unfamiliar culture also affect the health of family members and personal behaviors. Difficulties that foreign workers experience are very diverse, ranging from minor and usual things to structural problems, which may threaten their mental health and thus have a negative impact on their psychological wellbeing. Symptoms of depression or anxiety that occur in the process of cultural adaptation can be reduced during sports activities, and positive effects on mental health and satisfaction from life can be expected from the activities (Han and choi, 2011; Lee et al., 2013; Moon and Heo, 2015). Psychological wellbeing can be gained from pleasure from activities in daily life, positive self-image and self-esteem, and optimistic attitudes and emotions (Neugarten et al., 1961), and the Organisation for Economic Co-operation and Development (OECD) explains wellbeing with sat- isfaction of life, physical living conditions and quality of life (OECD Better Life Index, 2017). The psychological wellbeing of workers is a factor that determines the mental health of individuals, and improves not only their health, but also their work efficiency (Carolan et al., 2017), and also affects the productivity of businesses (Koo and Suh, 2013), having a significant impact on the economic development of countries. Meanwhile, one thing that needs to be considered before measuring the psychological wellbeing of foreign workers is the fact that wellbeing is easily affected by the distinct characteristics of culture (Jung et al., 2016).

Earlier studies conducted in Korea on the psychological wellbeing of foreign workers showed that Taekwondo practices improved their sense of accomplishment (Kim and Shin, 2018), and had a positive impact on psychological wellbeing (Yoon, 2011), and that improvements in the quality of life of their family in their motherland were recognized as their own quality of life (Lee, 2010), which was found to give a high level of happiness to them unlike the expectation of the researcher. It was difficult to find studies that were conducted in other countries on the psychological wellbeing of foreign workers for the past decade. There was one study on a similar variable, happiness, and $\mathrm{Li}$ et al. (2018) reported that psychological integration directly affected happiness (Jeong, 2019). However, there is almost no study that used forest healing programs to examine stress from cultural adaptation or psychological wellbeing. Against this backdrop, this study aimed to examine stress that foreign workers experience from cultural adaptation and their psychological wellbeing by applying a forest healing program, and to identify relationships between these variables, and the results of this study are expected to be utilized as basic data for reducing their stress from cultural adaptation and restoring their psychological wellbeing.

\section{Research Methods}

\section{Subjects}

The subjects of this study were a total to 40 foreign workers (16 males, 24 females) who were engaged in jobs related 
to agriculture in Hongcheon-gun, Gangwon-do. The treatment group of subjects participated in a forest-healing program held in a recreation forest located in Hongcheon-gun for three days and two nights, while the control group of subjects performed activities such as sowing the seeds of or harvesting vegetables such as lettuce, white radish, cabbage, chili, cucumber and pumpkin without participating in the program. The program was designed to compare changes in the conditions of subjects before and after the program by conducting pre and post-program tests. The subjects were selected among those who understood the purpose of objectives of this study, wanted to participate in this experiment and signed a written consent form, and the detailed criteria for selecting the subjects were as follows: those who were aged 20 or older; those who were not illegal aliens; those who could communicate in Korean; and those who fully understood the purpose of this study and consented to participate in the questionnaire survey for this study. The general characteristics of the subjects selected based on the criteria were as shown in Table 1.

The general characteristics of the subjects were divided into gender, age, educational background and nationality. The results of analyzing their general characteristics showed that the share of females $(24,60 \%)$ was higher than that of males $(15,40 \%)$. The number of those aged in their 20s-30s was $7(17,5 \%)$, those in their 30-40s, 18 (45\%); those in their 40-50, $13(32.5 \%)$; and those in their 50 s or older, $2(5 \%)$. In terms of academic background, 7 (17.5\%) out of the total subjects were 2-year college graduates or higher, 25 high school graduates (62.5\%), and 8 middle school graduates or below (20\%). Their nationality was also surveyed, and 32 came from Thailand, four from Vietnam and four from China. As shown in Table 1, the general characteristics of the treatment and control groups were similar. The foreign workers who participated in this experiment came from different countries, and their proficiency of Korean was very low. Those who fully understood the purpose and objectives of this program and consented to participate in the program were selected, and the program was led by a forest therapist.

\section{Procedure}

In this study, a forest healing program mainly composed of forest walking and meditation was provided in a recreation forest located in Gangwon-do for three days and two nights from June 26 to 28, 2019. A questionnaire survey was performed two times before and after providing the program using the same questionnaire form.

Table 1. Characteristics of participants $(\mathrm{N}=40)$

\begin{tabular}{|c|c|c|c|c|c|}
\hline \multirow{2}{*}{ Variable } & & \multicolumn{2}{|c|}{ Treatment group } & \multicolumn{2}{|c|}{ Control group } \\
\hline & & Frequency & Percent $(\%)$ & Frequency & Percent $(\%)$ \\
\hline \multirow{2}{*}{ Gender } & Male & 8 & 40 & 8 & 40 \\
\hline & Female & 12 & 60 & 12 & 60 \\
\hline \multirow{4}{*}{ Age } & $20-30$ & 3 & 15 & 4 & 20 \\
\hline & $30-40$ & 9 & 45 & 9 & 45 \\
\hline & $40-50$ & 7 & 35 & 6 & 30 \\
\hline & Over 50 years old & 1 & 5 & 1 & 5 \\
\hline \multirow{3}{*}{ Education } & Middle school graduate or lower & 2 & 10 & 6 & 30 \\
\hline & High school graduate & 13 & 65 & 12 & 60 \\
\hline & 2-year college graduate or higher & 5 & 25 & 2 & 10 \\
\hline \multirow{4}{*}{ Nationality } & Thailand & 16 & 80 & 16 & 80 \\
\hline & Vietnam & 2 & 10 & 2 & 10 \\
\hline & China & 2 & 10 & 2 & 10 \\
\hline & Total & 20 & 100 & 20 & 100 \\
\hline
\end{tabular}




\section{Measurement tools}

\section{Stress from cultural adaptation}

The scale to measure stress from cultural adaptation used in this study was the scale that was originally developed by Cervantes et al. (1991), was revised by Alderete et al. (1999) for immigrant workers, and was revised and adapted by Kim (2017) for Nepalese workers in Korea. The scale was divided into three sub-factors as follows: four questions about experience of discrimination; three questions about language conflicts; and six questions about legal status, a total of 13 questions. Each question was measured using a 5 -point Likert scale (1=strongly disagree; $5=$ strongly agree), and the higher the score, the higher the level of stress from cultural adaptation. Cronbach's $\alpha$ in Lee (2004) was .81 and that in this study was .85. The detailed content and Cronbach's $\alpha$ of the tool used to measure stress from cultural adaptation were as shown in Table 2 .

\section{Psychological wellbeing}

The scale of psychological wellbeing used in this study was the scale that was originally developed by Yang (1998) and was revised by Park and Kim (2010). The scale was divided into four sub-factors as follows: five questions about confidence; three questions about ego; three ques- tions about immersion; and three questions about joy, a total of 14 questions. Each questions was measured using a 5 -point Likert scale ( $1=$ strongly disagree; $5=$ strongly agree), and the higher the score, the higher the level of psychological wellbeing. The Cronbach's $\alpha$ of the original scale was .86 and that in this study was .83 (Table 3).

\section{Forest healing program}

\section{Composition and content of forest healing program}

Activities in the forest healing program used in this study were designed to give workers an opportunity to relax and recharge themselves in forest, to reduce their stress from cultural adaptation and to improve their psychological wellbeing through healing activities. The program was planned and led by a forest therapist, and the researchers of this study participated in the program as an assistant. The detailed activities were as shown in Table 4 .

\section{Activities of forest healing program}

On the first day of the program, an orientation was given to subjects, and they also signed a consent form and participated in the preliminary questionnaire survey. During the hours of drinking flower tea and self-introduction as an ice breaker, they were able to open themselves up and have

Table 2. Item composition of each sub-domain and the coefficient of Cronbach's $\alpha$ for stress measurement

\begin{tabular}{lcccc}
\hline \multicolumn{1}{c}{ Sub-factor } & Item count & Item number & \multicolumn{2}{c}{ Cronbach's $\alpha$} \\
\cline { 3 - 5 } & & & Kim (2017) & This study \\
\hline Discrimination experience & 4 & $1,5,9,13$ & .80 & .85 \\
Language conflict & 3 & $3,7,11$ & .84 & .86 \\
Legal status & 6 & $2,4,6,8,10,12$ & .79 & .84 \\
Total & 13 & 13 & .81 & .85 \\
\hline
\end{tabular}

Table 3. Item composition of each sub-domain and the coefficient of Cronbach's $\alpha$ for psychological well-being

\begin{tabular}{lcccc}
\hline \multirow{2}{*}{ Sub-factor } & Item count & Item number & \multicolumn{2}{c}{ Cronbach's $\alpha$} \\
\cline { 4 - 5 } & & & Park and Kim(2010) & This study \\
\hline Confidence & 5 & $5,7,6,8,4$ & .89 & .86 \\
Ego & 3 & $14,13,12$ & .90 & .91 \\
Immersion & 3 & $1,2,3$ & .82 & .80 \\
Joy & 3 & $10,9,11$ & .75 & .75 \\
Total & 14 & 14 & .86 & .83 \\
\hline
\end{tabular}


Table 4. Contents of the forest healing program

\begin{tabular}{|c|c|c|c|}
\hline Time & Day 1 & Day 2 & Day 3 \\
\hline 08:00 - 09:30 & & Breakfast & Breakfast \\
\hline 09:30 - 11:00 & & \multirow{2}{*}{ Forest walking and conversation 2} & Meditation 3 (walking) \\
\hline $11: 00-12: 00$ & & & Post-test \\
\hline $12: 00-13: 00$ & & Lunch & Lunch \\
\hline $13: 00-14: 00$ & & Free time & Checking out \\
\hline $14: 00-15: 00$ & Checking in and orientation & Meditation 2 (nature) & \\
\hline $15: 00-16: 00$ & Pre-test & Mandala & \\
\hline $16: 00-17: 00$ & Drinking flower tea & \multirow{2}{*}{ Forest walking and conversation 3} & \\
\hline $17: 00-18: 00$ & Free time & & \\
\hline $18: 00-19: 30$ & Forest walking and conversation 1 & Meditation 2 (forest) & \\
\hline $19: 30-20: 30$ & Dinner and free time & Dinner and free time & \\
\hline $20: 30-21: 00$ & Introducing yourself & Viewing stars in the night sky & \\
\hline $21: 00-22: 00$ & Meditation 1 (sleep) & Sharing your feelings & \\
\hline 22:00 - & Sleep & Sleep & \\
\hline
\end{tabular}

a chat. During the activity of Forest Walking 1, they had a chance to reflect on and understand themselves through body scanning, which was designed to help them establish their ego-identity. Sleep Meditation was designed to have a rest and meditate through physical and mental relaxation and breathing, and was composed of easy positions that beginners can easily follow, giving them a time for inner healing.

On the second day, activities such as Forest Walking 2 and 3, and Nature and Forest Meditation were performed, Forest Walking 2 and Nature Meditation were designed to silently look in their inner side and practice how to empty their mind. Mandara was a program of decorating natural materials in forest and find the inner me. Forest Meditation was a meditation activity for relaxation, and subjects lay on their back in forest to relax the whole body and have deep relaxation. The activity of looking at the night sky alone was designed to give subjects a time for looking back themselves.

On the third day, subjects had activities including Walking Meditation 3, wrapping up the program and the post-program questionnaire. Walk Meditation 3 was an activity of awakening the senses of the body by walking barefoot in forest, and self-examining by slowly walking and breathing.

\section{Data analysis}

The data collected in this study were analyzed using SPSS 18.0 Windows, a statistical analysis program, and frequency analysis was conducted to examine the general characteristics of subjects. The reliability of the collected data was analyzed using Cronbach's $\alpha$. The independent two sample t-test was conducted to verify the data collected before and after the forest healing program, and the paired t-test was conducted to verify average differences between the two variables measured from the same sample. The significance level was $p<.05$.

\section{Results and Discussion}

\section{Effects of forest healing program on stress from cultural adaptation}

To identify the level of stress from cultural adaptation, the values before and after participating in the forest healing program were compared, and the average statistics of the treatment group showed a statistically significant decrease. The scale used to measure the level of stress from cultural adaptation was composed of four questions about discrimination experience, three questions about language conflict and six questions about legal status, a total of 13 
questions (treatment group, pre-program $\mathrm{M}=2.60$, post-program $\mathrm{M}=1.78$; control group, pre-program $\mathrm{M}=2.51$, and post-program $\mathrm{M}=2.16$ ). The mean values and standard deviation values of the sub-factors before and after participating in the program were as shown in Table 5.

The results of each sub-factor were as follows: 4 questions about discrimination experience (treatment group, pre-program $\mathrm{M}=2.73$, post-program $\mathrm{M}=1.84$; control group, pre-program $\mathrm{M}=2.60$, post-program $\mathrm{M}=2.08$ ); 3 questions about language conflict (treatment group, pre-program $\mathrm{M}=2.61$, post-program $\mathrm{M}=1.86$; control group, pre-program $\mathrm{M}=2.45$, post-program $\mathrm{M}=2.20$ ); and 6 questions about legal status (treatment group, pre-program $\mathrm{M}=2.46$, post-program $\mathrm{M}=1.64$; control group, pre-program $\mathrm{M}=2.48$, post-program $\mathrm{M}=2.20$ ). Compared to the pre-program data, sub-factors including discrimination experience, legal status and language conflict showed statistically significant decreases after the program.

These results were similar to the results of Kim (2013) on the effects of leisure activities on the cultural adaptation of married immigrants, Jeong (2019) on the effects of stress from cultural adaptation and social support on the psychological wellbeing of foreign workers, and Lee (2004) that the effects of stress from cultural adaptation on the mental health of foreign workers. These earlier studies reported statistically significant differences in every surveyed sub-factor.

\section{Effects of forest healing program on psychological wellbeing}

To identify the psychological wellbeing of subjects, the values before and after participating in the forest healing program were compared, and statistically significant differences were observed. The scale used to measure the level of psychological wellbeing was composed of five questions about confidence, three questions about ego, three questions about immersion and three questions about joy, a total of 14 questions (treatment group, pre-program $\mathrm{M}=3.33$, post-program $\mathrm{M}=4.10$; control group, pre-program $\mathrm{M}=3.46$, post-program $\mathrm{M}=3.63$ ). The mean values and standard deviation values of the sub-factors before and after participating in the program were as shown in Table 6 .

To examine the psychological wellbeing of subjects, their data before and after participating in the program were compared, and the overall level of psychological wellbeing increased, showing statistically significant differences. The results of each sub-factor were as follows (Table 6): 5 questions about confidence (treatment group, pre-program $\mathrm{M}=3.19$, post-program $\mathrm{M}=4.00$; control group, pre-program $\mathrm{M}=3.32$, post-program $\mathrm{M}=3.59$ ); 3 questions about ego (treatment group, pre-program $\mathrm{M}=3.63$, post-program $\mathrm{M}=4.25$; control group, pre-program $\mathrm{M}=3.75$, post-program $\mathrm{M}=3.75$ ); 3 questions about immersion (treatment group, pre-program $\mathrm{M}=3.53$, post-program $\mathrm{M}=4.16$; control group, pre-program $\mathrm{M}=3.70$, post-program $\mathrm{M}=3.63$ ); and 3 questions about joy (treatment group, pre-program $\mathrm{M}=2.98$,

Table 5. Changes in cultural adaptation stress before and after the forest healing program

\begin{tabular}{|c|c|c|c|c|c|c|c|c|}
\hline \multirow{2}{*}{ Sub-factor } & \multirow{2}{*}{ Group } & \multicolumn{2}{|c|}{ Before } & \multicolumn{2}{|c|}{ After } & \multirow{2}{*}{$\mathrm{df}$} & \multirow{2}{*}{$\mathrm{t}$} & \multirow{2}{*}{$p$} \\
\hline & & M & $\mathrm{SD}$ & M & SD & & & \\
\hline \multirow{2}{*}{ Discrimination experience } & Treatment group & 2.73 & 0.35 & 1.84 & 0.34 & 38 & \multirow{2}{*}{-2.087} & \multirow{2}{*}{$.044^{*}$} \\
\hline & Control group & 2.60 & 0.37 & 2.08 & 0.38 & 38 & & \\
\hline \multirow{2}{*}{ Language conflict } & Treatment group & 2.61 & 0.46 & 1.86 & 0.80 & 38 & \multirow{2}{*}{-4.943} & \multirow{2}{*}{$.000 * *$} \\
\hline & Control group & 2.45 & 0.31 & 2.20 & 0.58 & 38 & & \\
\hline \multirow{2}{*}{ Legal status } & Treatment group & 2.46 & 0.40 & 1.64 & 0.24 & 38 & \multirow{2}{*}{-7.136} & \multirow{2}{*}{$.000 * *$} \\
\hline & Control group & 2.48 & 0.38 & 2.20 & 0.25 & 38 & & \\
\hline \multirow{2}{*}{ Total } & Treatment group & 2.60 & 0.24 & 1.78 & 0.18 & 38 & \multirow{2}{*}{-4.811} & \multirow{2}{*}{$.000^{* *}$} \\
\hline & Control group & 2.51 & 0.26 & 2.16 & 0.30 & 38 & & \\
\hline
\end{tabular}

Note. Treatment group, $\mathrm{n}=20$; control group, $\mathrm{n}=20$.

${ }^{*} p<.05,{ }^{*} p<.001$ 
Table 6. Changes in psychological well-being before and after the forest healing program

\begin{tabular}{|c|c|c|c|c|c|c|c|c|}
\hline \multirow{2}{*}{ Sub-factor } & \multirow{2}{*}{ Group } & \multicolumn{2}{|c|}{ Before } & \multicolumn{2}{|c|}{ After } & \multirow{2}{*}{$\mathrm{df}$} & \multirow{2}{*}{$\mathrm{t}$} & \multirow{2}{*}{$p$} \\
\hline & & $\mathrm{M}$ & $\mathrm{SD}$ & $\mathrm{M}$ & SD & & & \\
\hline \multirow{2}{*}{ Confidence } & Treatment group & 3.19 & 0.46 & 4.00 & 0.32 & 38 & \multirow{2}{*}{4.145} & \multirow{2}{*}{$.000^{* *}$} \\
\hline & Control group & 3.32 & 0.36 & 3.59 & 0.30 & 38 & & \\
\hline \multirow{2}{*}{ Ego } & Treatment group & 3.63 & 0.42 & 4.25 & 0.44 & 38 & \multirow{2}{*}{4.539} & \multirow{2}{*}{$.000^{* *}$} \\
\hline & Control group & 3.37 & 0.21 & 3.75 & 0.21 & 38 & & \\
\hline \multirow{2}{*}{ Immersion } & Treatment group & 3.53 & 0.38 & 4.16 & 0.62 & 38 & \multirow{2}{*}{3.348} & \multirow{2}{*}{$.002 *$} \\
\hline & Control group & 3.70 & 0.28 & 3.63 & 0.34 & 38 & & \\
\hline \multirow{2}{*}{ Joy } & Treatment group & 2.98 & 0.66 & 3.98 & 0.38 & 38 & \multirow{2}{*}{3.590} & \multirow{2}{*}{$.001^{* *}$} \\
\hline & Control group & 3.06 & 0.41 & 3.53 & 0.41 & 38 & & \\
\hline \multirow{2}{*}{ Total } & Treatment group & 3.33 & 0.48 & 4.10 & 0.44 & 38 & \multirow{2}{*}{7.010} & \multirow{2}{*}{$.000 * *$} \\
\hline & Control group & 3.46 & 0.32 & 3.63 & 0.32 & 38 & & \\
\hline
\end{tabular}

Note. Treatment group, $\mathrm{n}=20$; control group, $\mathrm{n}=20$.

$* p<.05, * * p<.001$.

post-program $\mathrm{M}=3.98$; control group, pre-program $\mathrm{M}=3.06$, post-program $\mathrm{M}=3.53$ ). The control group showed a decrease in immersion after participating in the program, but the experiment group showed statistically significant differences in every sub-factor including confidence, ego, immersion and joy. These results coincided with the results of Park (2003), a comparison study on the effects of working conditions for foreign and Korean workers on their satisfaction from work; Park (2012) on the structural relations of sense of accomplishment, psychological wellbeing and intention to continue to participate depending on the image of aerobic dance instructors (Park, 2012); and Lee (2004) on the effects of stress from cultural adaptation on the mental health of foreign workers. These earlier studies reported statistically significant differences in every surveyed sub-factor. Although the tools for mediation used in these studies differ, they all showed positive results. As such, the forest healing program is also expected to be a very effective tool for mediation.

\section{Conclusion}

In this study, a 3-day, 2-night forest healing program focusing on forest walking and meditation was provided for foreign workers in order to identify the effects of the program on their stress from cultural adaptation and psy- chological wellbeing. Based on the results of this study, the following conclusions were reached. First, the effects of the forest healing program on their stress from cultural adaptation were examined, and it was found that the lower the level of stress from cultural adaptation, the higher the level of psychological wellbeing. Among the sub-factors surveyed in this study, the treatment group showed statistically significant differences in discrimination experience compared to the control group $(p<.05)$, and the differences in language conflict and legal status were very statistically significant $(p<.001)$. The results can be attributed to the opportunities that the program provided for subjects to freely express their feelings and thoughts. The process of asking who I am and finding themselves through activities including touching trees and lying back to look at the sky in forest, and meditation in nature seemed to positively affect their stress from cultural adaptation. Second, while the control group showed a decrease in psychological wellbeing after the program ( $\mathrm{M}=3.70$ to 3.63$)$, the treatment group showed statistically significant differences in all the sub-factors surveyed including confidence, ego, immersion and joy. Opportunities to look back about themselves through activities such as looking at the night sky alone, break ice between participants through self-introduction, and share their feelings they felt living in Korea seemed to promote to build sympathy and support between foreign workers, and bond and sympathy with fellow workers. In 
addition, words of encouragement and support seemed to draw positive energy and improve their adaptability to the reality, improving psychological wellbeing. Third, the forest healing program focusing on forest walking and meditation reduced stress from cultural adaptation and improve psychological wellbeing. In order to adapt themselves to a different society and culture, communication is essential, and the reason why these positive results were obtained above was that they were able to break ice, feel a sense of closeness and build bond between participants through several activities before participating in the program, which seemed to create rapport between them. They were able to get to know each other by themselves even during the program, and to have chances to share and frankly speak about the difficulties they have experienced, which seemed to make them understand each other better. Since the program was performed for 3-days and 2-nights, a limited amount of time, it is difficult to generalize the results of this study. Therefore, it will be necessary to conduct more in-depth studies going forward to address this limitation.

Based on the conclusions above, the following suggestions can be presented. First, since the period of this study was only 3-days and 2-nights, which is relatively short, and how long the effects lasted after the program was not surveyed, it is necessary to conduct a follow-up study on the long-term effects of the program. Second, it is also needed to compare the effects on the cultural adaptation stress and psychological wellbeing of foreign workers with those of Korean workers. Third, it is necessary to develop a program that can reduce the stress that foreign workers experience from cultural adaptation and improve their psychological wellbeing considering their social support, health conditions and academic background. The results of this study are expected to be utilized as basic data for further identifying the effects of forest healing programs focusing on forest walking and meditation going forward.

\section{References}

Alderete, E., W.A. Vega, B. Kolody, and S. Aguilar-Gaxiola. 1999. Depressive symptomatology: Prevalence and psychosocial risk factors among Mexican migrant farmworkers in California. J. Community Psychol. 27(4):457-471.

Berry, J.W. 1984. Cultural relations in plural societies: Alternatives to segregation and their sociopsychological implications. In N.Miller and M. Brewer (Eds.), Groups in contact. New York: Academic Press.

Berry, J.W. 1997. Immigration, acculturation, and adaptation. Appl. Psychol. 46(1):5-34. https://doi.org/10.1111/j.14 64-0597.1997.tb01087.x

Berry, J.W., U. Kim, T. Minde and D. Mok. 1987. Comparative studies of acculturative stress. Int. Migr. Rev. 21(3):491-511. https://doi.org/10.2307/2546607

Carolan, S., P.R. Harris, and K. Cavanagh. 2017. Improving employee well-being and effectiveness: Systematic review and meta-analysis of web-based psychological interventions delivered in the workplace. J. Med. Internet Res. 19(7):e271. https://doi.org/10.2196/jmir.7583

Cervantes, R.C., A.M. Padilla, and N. Salgado de Snyder. 1991. The hispanic stress inventory: A culturally relevant approach to psychosocial assessment. Psychol. Assess. 3(3):438-447. http://dx.doi.org/10.1037/1040-3590.3.3.438

Han, H.S. and M.O. Choi. 2011. A study of the social problems and policy response due to the increase of foreigners in Korea. GRI Res. Rev. 13(3):61-84. Retrieved from http://www.gri.re.kr

Hendriks, M. and D. Bartram. 2016. Macro-conditions and immigrants' happiness: Is moving to a wealthy country all that matters? Soc. Sci. Res. 56:90-107. https://doi.org/10.1016/j.ssresearch.2015.11.006

Jeong, H.N. 2019. The effect of acculturative stress and social support on psychological well-being of migrant workers. Master's thesis, Kosin University, Busan, Korea.

Jung, S.H., Y.J. Kim, and Y.S. Song. 2016. Effect of organizational culture on subjective well-being in organization. J. Korea Contents Assoc. 16(5):497-508.

Kim, E.J. 2013. Leisure activity of married immigrant women influencing cultural adjustment. : Focusing on moderating effects by language proficiency and language use. Doctoral dissertation, Sejong University, Seoul, Korea.

Kim, J.A. and I.K. Kim. 2011. Acculturative stress, social 
support and self efficacy for Chinese students in Korea. J. Korean Acad. Soc. Nurs. Edu. 17(1):52-61.

Kim, J.K. 2017. A study on the effect of resilience on the acculturation stress among Nepalese migrant workers in South Korea. Ment. Health [Seoul Mental Health Center] 7(1):22-33.

Kim, J.M. and J.S. Shin. 2018. The effects of self accomplishment found through Taekwondo training on the psychological happiness of foreign laborers living in Korea. J. World Soc. Taekwondo Cult. 9(1):79-97.

Kim, K.W. 2012. A study on the actual conditions and policy tasks of Korean multicultural families. Korean J. 21 Century Soc. Welf. 9(2):31-60.

Koo, J.S. and E.K. Suh. 2013. The influence of happiness on future income and job performance. Korean J. Soc. Pers. Psychol. 27(2):17-36. https://doi.org/10.21193/kjspp.2013.27.2.002

Korean National Police Agency. 2016. Crime statistics. Retrieved from https://www.police.go.kr

Lee, I.S. 2004. The influence of acculturative stress on migrant workers' mental health: focused on the moderating effects of social relationships. Master's thesis, Yonsei University, Seoul, Korea.

Lee, J.Y. 2010, August. Migrant worker's quality of life and their happiness in Korea. Proceedings of annual conference of Korean Psychological Association (pp. 298-299). Seoul, Korea: KPA.

Lee, S.H., Y.J. Lee, S.Y. Kim, and S.J. Kim. 2009. Social support and acculturative stress in migrant workers. J. Korean Acad. Nurs. 39(6):899-910. https://doi.org/10.4040/jkan.2009.39.6.899

Lee, S.L., Y.S. Kim, and S.S. Shim. 2013. Relationship among cultural adaptation stress, depression and life satisfaction of marriage migrant women in leisure sports participants. J. Leis. Recreat. Stud. 37(4):57-72.

Li, T.C., C.C. Chu, F.C.Meng, Q. Li, D. Mo, B. Li, and S.B. Tsai. 2018. Will happiness improve the psychological integration of migrant workers? Int. J. Environ. Res. Public Health 15(5):900. https://doi.org/10.3390/ijerph15050900
Liu, B. and W. Zhang. 2015. The happiness difference between two Chinese worker groups: A comparison between migrant workers on production line and local workers in railway electrical supply section. Procedia Manuf. 3:2281-2287. https://doi.org/10.1016/j.promfg. 2015.07.373

Moon, H.S. and H.M. Heo. 2015. The effects of sports participation on social adjustment and life satisfaction by foreign immigrant workers. Korean J. Sociol. Sport 28(2):65-85. https://doi.org/10.22173/jksss.2015.28.2.65

Neugarten, B.L., R.J. Havighurst, and S.S. Tobin. 1961. The measurement of life satisfaction. J. Gerontol. 16(2):134-143. https://doi.org/10.1093/geronj/16.2.134

OECD Better Life Index. 2017. Multilingual summaries how's life? 2017, Measuring well-being. Retrieved November 15, 2017 from http://www.oecdbetterlifeind ex.org/media/bli/documents/how life-2017-sum-en.pdf

Park, C.Y. 2012. A structural relationship between self-fulfillment, psychological happiness and participation duration intention by image of aerobic dance instructor. Korean J. Sport Psychol. 23(3):47-60.

Park, D.S. 2003. A comparative study on the effects of working conditions of foreign workers and Korean workers on job satisfaction. Master's thesis, Yonsei University, Seoul, Korea.

Park, I.S. and Y.H. Kim. 2010. The impact of line dance club members' participation on behaviors to improve health and psychological happiness: Targeting middle-aged women. J. Korean Phys. Educ. Assoc. Girls Women 24(5):57-70.

Park, K.K. and Y.K. Lim. 2000. A study of causal model between social stress, the incidence of crime and accidents. Korean J. Soc. Sci. 19(2):81-97.

Yang, M.H. 1998. Development of cognitive-affective states scale: Physical activity and psychological well-being. Korean J. Sport Psychol. 9(2):113-123.

Yoon, Y.H. 2011. Study on psychological well-being of foreign workers in Korea: Mainly of Chinese and Korean-Chinese workers. Master's thesis, Hannam University, Daejeon, Korea. 